\title{
Performance and complexity analysis of suboptimal MIMO detectors
}

\author{
Berna Özbek \\ Izmir Institute of Technology \\ Electrical and Electronics Engineering \\ Izmir, Turkey \\ Email: bernaozbek@iyte.edu.tr
}

\author{
Didier Le Ruyet \\ Conservatoire National des Arts et Métiers (CNAM) \\ Electronique et Communication \\ Paris, France \\ Email: leruyet@cnam.fr
}

\begin{abstract}
In this paper, we consider coded MIMO systems using suboptimal iterative receivers. We propose to use the list type breadth first detectors based on $M$ and TM algorithms and the soft output linear MMSE detectors with a priori information. We compare these two families of suboptimal iterative receivers in terms of complexity and bit-error-rate performance.
\end{abstract}

\section{INTRODUCTION}

The development of multiple-input multiple-output (MIMO) communication systems that use multiple transmit and multiple receive antennas provides the ability of designing spectrally efficient systems without extra power and bandwidth. It has been shown that the channel capacity grows linearly as the number of transmit and receive antennas grow simultaneously [1]. Many researches on MIMO systems have been performed to exploit the available capacity. In particular, the Vertical-Bell Laboratories Layered Space-Time (V-BLAST) can achieve the theoretically proven linear capacity growth with a simplified structure [2].

In order to achieve near-capacity performance, MIMO systems should be concatenated with outer channel codes such as a convolutional code (CC) or turbo code (TC) [3]. Although the classical detector for the V-BLAST scheme is based on nulling and canceling (NC) algorithm, for the coded V-BLAST systems, it is important to use an a posteriori probability (APP) detector for MIMO detection in an iterative manner to approach the performance of ideal joint receivers. It is well known that the computational complexity of a full APP detector increases with the number of transmit and receive antennas and the size of constellations. Therefore, the reduced complexity MIMO detectors, which can extract and/or exchange soft information required by outer channel decoders while providing a good approximation of full APP decoding, are needed [4].

In this paper, we propose to compare two families of suboptimal APP MIMO detectors: the soft-output minimum mean square error (MMSE) detector with a priori information [5], [6] and the list type breadth first $M$ and $T M$ algorithms [7].

This paper is organized as follows. In section 2, the linear system model of MIMO channels is described. The two suboptimal APP detectors for coded MIMO systems are proposed in section 3. Then, the complexity evaluations of these two suboptimal detectors are derived in section 4. Finally, the comparison results are given in terms of performance and complexity.

\section{LINEAR MODEL FOR MIMO CHANNEL}

We consider coded V-BLAST over flat fading MIMO channels with $N_{t}$ transmit and $N_{r}$ receive antennas as illustrated in Figure 1.

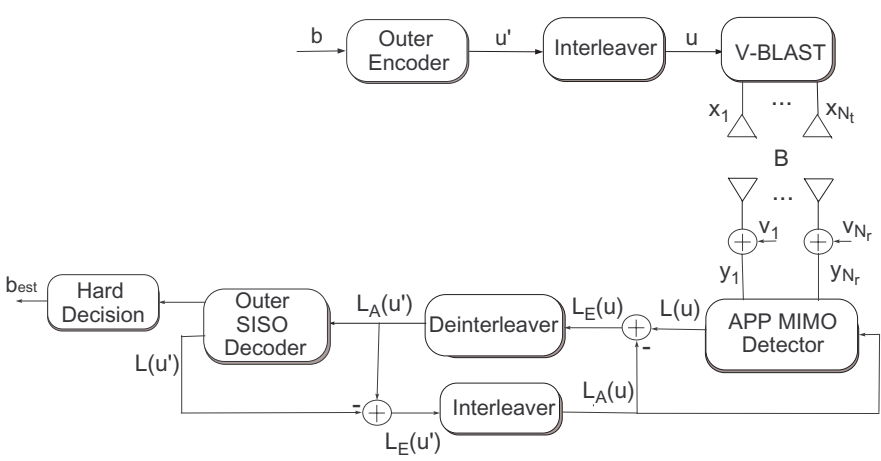

Fig. 1. The linear system model for coded V-BLAST

The vector of information bits $\mathbf{b}$ is first encoded with an error correcting code and then interleaved to obtain the vector of coded bits. Then, we decompose this vector into blocks of coded bits of length $2 N_{t} \times M_{c}, \mathbf{u}=\left(\mathbf{u}_{1}, \ldots, \mathbf{u}_{2 N_{t}}\right)^{T}$ with $\mathbf{u}_{p}=\left(u_{p 1}, \ldots, u_{p M_{c}}\right)$ where $M_{c}$ is the number of bits per real symbol. Each vector $\mathbf{u}$ is then mapped to the vector of real symbols $\mathbf{x}=\left(x_{1}, \ldots, x_{2 N_{t}}\right)^{T} \in \Lambda_{r}^{2 N_{t} \times 1}$. We suppose that $\Lambda_{r}$ is the $2^{M_{c}}$-PAM signal set $\Lambda_{r}=\left\{-2^{M_{c}}+\right.$ $\left.1,-2^{M_{c}}+3, \ldots, 2^{M_{c}}-3,2^{M_{c}}-1\right\}=\left\{\lambda_{1}, \lambda_{2}, \ldots, \lambda_{2^{M_{c}}}\right\}$ where $\lambda_{s} \in R$ corresponds to the bit pattern $\mathbf{b}_{s}=$ $\left(b_{s 1}, b_{s 2}, \ldots, b_{s M_{c}}\right)$. In other words, the complex symbols are chosen from a QAM constellation with $2^{2 M_{c}}$ possible signal points.

Then, we obtain the classical real-valued equation

$$
\mathbf{y}=\mathbf{B x}+\mathbf{v}
$$

where $\mathbf{B}$ is the real channel matrix of dimension $2 N_{r} \times 2 N_{t}$ built from the complex channel matrix $\mathbf{H}$ by replacing its 
elements $h_{i j}$ by $\left[\begin{array}{cc}\Re\left(h_{i j}\right) & -\Im\left(h_{i j}\right) \\ \Im\left(h_{i j}\right) & \Re\left(h_{i j}\right)\end{array}\right]$. The $h_{i j}$ is defined as the complex channel coefficient between the $i$ th transmit antenna and the $j$ th receive antenna and is modeled as an independent realization of complex Gaussian random variable of unit variance. $\mathbf{v}$ is a vector of independent zero mean real Gaussian noise with variance $\sigma_{n}^{2}$.

$\mathbf{x}$ is obtained from the vector of complex symbols $\mathbf{s}=$ $\left(s_{1}, \ldots, s_{N_{t}}\right)^{T}$ as $\mathbf{x}=\left(x_{1}, x_{2}, x_{3}, x_{4} \ldots, x_{2 N_{t}-1}, x_{2 N_{t}}\right)^{T}=$ $\left(\alpha_{1}, \beta_{1}, \alpha_{2}, \beta_{2} \ldots, \alpha_{N_{t}}, \beta_{N_{t}}\right)^{T}$ where $\alpha_{q}=\Re\left(s_{q}\right)$ and $\beta_{q}=$ $\Im\left(s_{q}\right)$. We assume that the energy of each real symbol $x_{p}$ is $\mathrm{E}\left\|x_{p}\right\|^{2}=\sigma_{x}^{2}=\frac{1}{2 N_{t}}$ and consequently the total transmit power per channel is $E_{s}=1$.

At the receiver with perfect channel knowledge, we consider an iterative system that consists of a MIMO detector and an outer soft input soft output decoder operating according to the log-MAP algorithm. The MIMO detector takes both channel and a priori information and then generates a posteriori information about the coded bits. After deinterleaving the an extrinsic information, an outer decoder generates decisions about the information bits as well as an extrinsic information about the coded bits. This information is fed back to the MIMO detector where it is used as a priori information.

\section{Suboptimal APP MiMO detectors}

Considering the system model given in (1), the APP of the transmitted bit $u_{p m}$ given the received vector $\mathbf{y}$ is usually expressed as a log-likelihood ratio (LLR) by

$L\left(u_{p m} \mid \mathbf{y}\right)=\ln \frac{\mathrm{P}\left(u_{p m}=+1\right)}{\mathrm{P}\left(u_{p m}=-1\right)}+\ln \frac{\sum_{\mathbf{u} \in \mathbb{U}_{p m,+1}} \mathrm{p}(\mathbf{y} \mid \mathbf{u}) \mathrm{P}\left(\mathbf{u} \mid u_{p m}\right)}{\sum_{\mathbb{U}_{p m,-1}} \mathrm{p}(\mathbf{y} \mid \mathbf{u}) \mathrm{P}\left(\mathbf{u} \mid u_{p m}\right)}$

where $p=1, \ldots, 2 N_{t}, m=1, \ldots, M_{c}$ and $\mathbb{U}_{p m,+1}$ is the set of $2^{\left(2 N_{t} M_{c}-1\right)}$ vectors $\mathbf{u}$ with $u_{p m}=+1$. The first term is the a priori $\mathrm{L}$-value, $L_{A}$, and the second term is the extrinsic L-value, $L_{E}$, that will be passed to the next decoder.

Using the max-log approximation and assuming the independence of bits, the LLR can be expressed as

$$
\begin{aligned}
L\left(u_{p m} \mid \mathbf{y}\right) & =\max _{\mathbf{u} \in \mathbb{U}_{p m,+1}}\left\{-\frac{\|\mathbf{y}-\mathbf{B} \mathbf{x}(\mathbf{u})\|^{2}}{2 \sigma_{n}^{2}}+P_{x}\right\} \\
& -\max _{\mathbf{u} \in \mathbb{U}_{p m,-1}}\left\{-\frac{\|\mathbf{y}-\mathbf{B x}(\mathbf{u})\|^{2}}{2 \sigma_{n}^{2}}+P_{x}\right\}
\end{aligned}
$$

with $P_{x}=\sum_{(a b)} \ln \mathrm{P}\left(u_{a b}\right)$ where $\ln \mathrm{P}\left(u_{a b}\right)$ can be calculated from the a priori information.

The output of the APP MIMO detector is obtained as an extrinsic L-value which is calculated by $L_{E}\left(u_{p m} \mid \mathbf{y}\right)=$ $L\left(u_{p m} \mid \mathbf{y}\right)-L_{A}\left(u_{p m}\right)$.

Computing of $L_{E}\left(u_{p m} \mid \mathbf{y}\right)$ is exponential in the length of the symbol vector $\mathbf{x}$ and the number of bits in the constellation. In order to find the maximum value of (3) for each bit, there are $2^{2 N_{t} M_{c}-1}$ possibilities to search over in each of the two terms. For example, if we calculate the LLR for $(8,8)$ V-BLAST with 16QAM constellation, we have $2^{2 \times 8 \times 2-1}=2^{31} \approx 2 \times 10^{9}$ possibilities.

\section{A. Breadth first $M$ and $T M$ algorithms}

Since the computing of (3) is of prohibitive complexity, a list of $M_{b}$ points that makes $\|\mathbf{y}-\mathbf{B x}(\mathbf{u})\|^{2}$ smallest is used [4]. Therefore, the list contains only the sequences $\mathbf{x}$ for which the metric

$$
\mathcal{M}(\mathbf{x}(\mathbf{u}))=\|\mathbf{Y}-\mathbf{B} \mathbf{x}(\mathbf{u})\|^{2}-2 \sigma_{n}^{2} \sum_{p=1}^{2 N_{t}} \ln P\left(x_{p}\right)
$$

is small.

In [4], the authors have shown that $\|\mathbf{y}-\mathbf{B x}(\mathbf{u})\|^{2}$ can be written in the following way

$$
\|\mathbf{y}-\mathbf{B x}(\mathbf{u})\|^{2}=(\mathbf{x}-\hat{\mathbf{x}})^{T} \mathbf{B}^{T} \mathbf{B}(\mathbf{x}-\hat{\mathbf{x}})+\mathcal{M}^{\prime}
$$

where $\mathcal{M}^{\prime}=\mathbf{y}^{T}\left(\mathbf{I}-\mathbf{B}\left(\mathbf{B}^{T} \mathbf{B}\right)^{-1} \mathbf{B}^{T}\right) \mathbf{y}$ and $\hat{\mathbf{x}}=$ $\left(\mathbf{B}^{T} \mathbf{B}\right)^{-1} \mathbf{B}^{T} \mathbf{y}$ is the unconstrained least squares (LS) estimate.

Assuming $2 N_{t} \leq 2 N_{r}$ and $\operatorname{rank}(\mathbf{B})=2 N_{t}$ and using (5), the metric in (4) is calculated for APP detection as

$$
\mathcal{M}(\mathbf{x}(\mathbf{u}))=\mathbf{z}^{T} \mathbf{P}^{T} \mathbf{P} \mathbf{z}-2 \sigma_{n}^{2} \sum_{p=1}^{2 N_{t}} \ln \mathrm{P}\left(x_{p}\right)+\mathcal{M}^{\prime}
$$

where $\mathbf{x}=\hat{\mathbf{x}}+\mathbf{z}$ is a candidate vector and $\mathbf{P}$ is the upper triangular matrix with $\mathbf{B}^{T} \mathbf{B}=\mathbf{P}^{T} \mathbf{P}$ obtained using the Cholesky factorization.

Let $P_{p s}$ be an element of the matrix $\mathbf{P}$ with $p, s \leq 2 N_{t}$ and $\mathbf{z}=\left(z_{1}, z_{2}, \ldots, z_{2 N_{t}}\right)^{T}$.

Using the exact expression and substituting $q_{p p}=P_{p p}^{2}$ and $q_{p s}=P_{p s} / P_{p p}$ for $p=1, \ldots, 2 N_{t}, s=p+1, \ldots, 2 N_{t}$, we can write

$\mathcal{M}(\mathbf{x}(\mathbf{u}))=\sum_{p=1}^{2 N_{t}}\left(q_{p p}\left(z_{p}+\sum_{s=p+1}^{2 N_{t}} q_{p s} z_{s}\right)^{2}-2 \sigma_{n}^{2} \ln \mathrm{P}\left(x_{p}\right)\right)+\mathcal{M}^{\prime}$

Since these algorithms can be seen as tree search algorithms, each path in the tree corresponds to a vector $\mathbf{x}$ and each branch of the tree can be labeled with a branch metric. For a branch at depth $p$, the branch metric $w\left(\mathbf{x}_{p}^{2 N_{t}}\right)$ is given by

$$
w\left(\mathbf{x}_{p}^{2 N_{t}}\right)=q_{p p}\left(z_{p}+\sum_{s=p+1}^{2 N_{t}} q_{p s} z_{s}\right)^{2}-2 \sigma_{n}^{2} \ln \mathrm{P}\left(x_{p}\right)
$$

where $\mathbf{x}_{p}^{2 N_{t}}=\left(x_{p}, x_{p+1} \ldots, x_{2 N_{t}}\right)^{T}$ is the vector associated with the path from the root of the tree to the branch. Then we may write the accumulated metric corresponding to this partial path in the form as

$$
\begin{aligned}
\mathcal{M}\left(\mathbf{x}_{p}^{2 N_{t}}\right) & =\sum_{s=p}^{2 N_{t}} w\left(\mathbf{x}_{s}^{2 N_{t}}\right)+\mathcal{M}\left(\mathbf{x}_{2 N_{t}+1}^{2 N_{t}}\right) \\
& =w\left(\mathbf{x}_{p}^{2 N_{t}}\right)+\mathcal{M}\left(\mathbf{x}_{p+1}^{2 N_{t}}\right)
\end{aligned}
$$

with $\mathcal{M}^{\prime}=\mathcal{M}\left(\mathbf{x}_{2 N_{t}+1}^{2 N_{t}}\right)$ is the initial value and $w\left(\mathbf{x}_{s}^{2 N_{t}}\right)$ is the $s$ th branch metric.

We use the breadth first $M$ algorithm in order to obtain the list of candidates as proposed in [7]. The main idea is that the survivors with small weight can be omitted with a 
negligible probability of discarding the most likelihood paths. At each depth while the $M$ algorithm keeps a fixed number of paths $\left(M_{b}\right)$, the $T M$ algorithm keeps a variable number of survivor paths depending on a chosen threshold parameter. This number is limited to $M_{b}$ in order to limit the memory size and the computational complexity.

Depending on the chosen center of the search such as the received point $\mathbf{y}$, the maximum likelihood (ML) point or the Babai (NC) point, we can derive different versions of the $T M$ algorithm. In this paper, we only consider the last version since it gives better performance than using the received point and avoids a precomputation of the center like for the ML point case. This metric condition is given by

$$
\mathcal{M}\left(\mathbf{x}_{p}^{2 N_{t}}\right)-\mathcal{M}\left(\mathbf{x}_{p}^{2 N_{t}}\right)_{\min } \leq C_{1}
$$

where $\mathcal{M}\left(\mathbf{x}_{p}^{2 N_{t}}\right)_{\min }$ corresponds to the path obtained using the $\mathrm{NC}$ algorithm. Then, the $\mathrm{NC}$ point is selected as the reference point in lattice for starting the search.

In comparison with the list sphere decoder (LSD) algorithm, the breadth first algorithms are performed from the root to the leaves of the tree. In a hardware realization, the memory size of the decoder for the list is limited. In this case, LSD requires to perform the sorting and enumeration operations and then eliminates the worse candidate at each visited point. However, $T M$ detector does the sorting and enumeration at each depth. This decreases the complexity of $T M$ detector significantly compared to LSD.

For the breadth first $M$ and $T M$ algorithms using only the selected paths according to their metrics, the LLR in (3) can be written as

$$
\begin{aligned}
L\left(u_{p m} \mid \mathbf{y}\right) & =\max _{\mathbf{u} \in \mathbb{V}_{p m,+1}}\left\{-\frac{1}{2 \sigma_{n}^{2}} \mathcal{M}(\mathbf{x}(\mathbf{u}))\right\} \\
& -\max _{\mathbf{u} \in \mathbb{V}_{p m,-1}}\left\{-\frac{1}{2 \sigma_{n}^{2}} \mathcal{M}(\mathbf{x}(\mathbf{u}))\right\}
\end{aligned}
$$

where $\mathbb{V}_{p m,+1}$ is the set of $M_{b}$ selected vectors $\mathbf{u}$ with $u_{p m}=$ +1 .

\section{B. Soft output MMSE detector with a priori information}

A suboptimal soft input soft output receiver based on soft interference cancellation and linear MMSE filtering have been proposed in [5]. The MMSE equalization techniques using a priori information which enables significant reduction in computational complexity of turbo equalization methods have been discussed in [8]. The soft output MMSE detector using a priori information for MIMO systems has been proposed in [6].

Instead of computing (2), soft input soft output MMSE detector first computes an affine estimate $\hat{\mathbf{x}}$ from the observation $\mathbf{y}$ in (1)

$$
\hat{\mathbf{x}}=\mathbf{A y}+\mathbf{b}
$$

where $\mathbf{A}=\operatorname{cov}(\mathbf{x}, \mathbf{y}) \operatorname{cov}(\mathbf{y}, \mathbf{y})^{-1}$ and $\mathbf{b}=E(\mathbf{x})-\mathbf{A B} E(\mathbf{x})$ which are obtained by minimizing the mean square error (MSE) between $\mathbf{x}$ and $\hat{\mathbf{x}}\left(\|\mathrm{x}-\hat{\mathbf{x}}\|^{2}\right)$.
Since $\operatorname{cov}(\mathbf{y}, \mathbf{y})=\left(\mathbf{B} \operatorname{cov}(\mathbf{x}, \mathbf{x}) \mathbf{B}^{T}+\sigma_{n}^{2} \mathbf{I}\right)^{-1}$ and $\operatorname{cov}(\mathbf{x}, \mathbf{y})=\operatorname{cov}(\mathbf{x}, \mathbf{x}) \mathbf{B}^{T}$, finally we get the MMSE solution as

$\hat{\mathbf{x}}=E(\mathbf{x})+\operatorname{cov}(\mathbf{x}, \mathbf{x}) \mathbf{B}^{T}\left(\mathbf{B} \operatorname{cov}(\mathbf{x}, \mathbf{x}) \mathbf{B}^{T}+\sigma_{n}^{2} \mathbf{I}\right)^{-1}(\mathbf{y}-\mathbf{B} E(\mathbf{x}))$

$\mathrm{E}(\mathbf{x})$ and $\operatorname{cov}(\mathbf{x}, \mathbf{x})$ are estimated using the a priori information $L_{A}\left(u_{p m}\right)$ on the bits forming the symbols of $\mathbf{x}$. As given in [8], the expectation of $x_{p}$ is

$$
\mathrm{E}\left(x_{p}\right)=\sum_{\lambda_{s} \in \Lambda_{r}} \lambda_{s} \mathrm{P}\left(x_{p}=\lambda_{s}\right)
$$

Since the bits are independent, $\operatorname{cov}\left(x_{p}, x_{s}\right)=0$ for all $p$ and $s$ where $p \neq s$. Then, as given in [8], the diagonal terms are

$$
\operatorname{cov}\left(x_{p}, x_{p}\right)=\left(\sum_{\lambda_{s} \in \Lambda_{r}}\left|\lambda_{s}\right|^{2} \mathrm{P}\left(x_{p}=\lambda_{s}\right)\right)-\left|\mathrm{E}\left(x_{p}\right)\right|^{2}
$$

Then, the extrinsic information on the bits of the symbol $x_{p}$ can be produced from the soft estimate of symbol. For iterative process, it is known that a priori information on these bits should not be used to produce the soft estimate of a symbol. Therefore, (14) should be separated into $2 N_{t}$ equations, one per real dimension, where the estimate on each real dimension does not use the a priori statistics of the associated symbol. We denote $\mathrm{E}_{p}(\mathrm{x})$ as the expectation with respect to the input symbols' statistics not using the a priori information on the bits of $x_{p}$. Similarly, $\operatorname{cov}_{p}(\mathbf{x}, \mathbf{x})$ is the covariance of $\mathbf{x}$ not using the a priori information on the bits of $x_{p}$. Then, for $q=1,2, \ldots, N_{t},(14)$ can be written as

$$
\begin{aligned}
\hat{\alpha}_{q} & =\frac{\sigma_{x}^{2}}{2} \mathbf{B}_{2 q-1}^{T} \operatorname{cov}_{q}^{-1}\left(\mathbf{y}-\mathbf{B E}_{q}(\mathbf{x})\right) \\
\hat{\beta}_{q} & =\frac{\sigma_{x}^{2}}{2} \mathbf{B}_{2 q}^{T} \operatorname{cov}_{q}^{-1}\left(\mathbf{y}-\mathbf{B E}_{q}(\mathbf{x})\right)
\end{aligned}
$$

where $\mathbf{B}_{q}$ is the $q$ th column of $\mathbf{B}$,

$$
\operatorname{cov}_{q}=\mathbf{B}_{\operatorname{cov}_{q}}(\mathbf{x}, \mathbf{x}) \mathbf{B}^{T}+\sigma_{n}^{2} \mathbf{I}_{2 N_{t}}
$$

and

$$
\begin{aligned}
\mathrm{E}_{q}(\mathbf{x})= & \left(\mathrm{E}\left(\hat{\alpha}_{1}\right), \mathrm{E}\left(\hat{\beta}_{1}\right), \ldots, \mathrm{E}\left(\hat{\beta}_{q-1}\right),\right. \\
& \left.0,0, \mathrm{E}\left(\hat{\alpha}_{q+1}\right), \ldots, \mathrm{E}\left(\hat{\alpha}_{N_{t}}\right), \mathrm{E}\left(\hat{\beta}_{N_{t}}\right)\right)^{T} \\
\operatorname{cov}_{q}(\mathbf{x}, \mathbf{x})= & \operatorname{diag}\left(\operatorname{cov}\left(\hat{\alpha}_{1}\right), \operatorname{cov}\left(\hat{\beta}_{1}\right), \ldots, \operatorname{cov}\left(\hat{\beta}_{q-1}\right)\right. \\
& \left.\frac{\sigma_{x}^{2}}{2}, \frac{\sigma_{x}^{2}}{2}, \operatorname{cov}\left(\hat{\alpha}_{q+1}\right), \ldots, \operatorname{cov}\left(\hat{\alpha}_{N_{t}}\right), \operatorname{cov}\left(\hat{\beta}_{N_{t}}\right)\right)^{T}
\end{aligned}
$$

Following [5], we assume that $\hat{\alpha}_{q}$ and $\hat{\beta}_{q}$ are the outputs of equivalent Gaussian channels and we can write

$$
\begin{aligned}
& \hat{\alpha}_{q}=\mu_{2 q-1} \alpha_{q}+\eta_{2 q-1} \\
& \hat{\beta}_{q}=\mu_{2 q} \beta_{q}+\eta_{2 q}
\end{aligned}
$$

where $\mu_{2 q-1}$ is the gain of the equivalent Gaussian channels and $\eta_{2 q-1}$ is the associated noise with variance $\nu_{2 q-1}^{2}$. These values are calculated as

$$
\begin{aligned}
\mu_{2 q-1} & =\frac{\sigma_{x}^{2}}{2} \mathbf{B}_{2 q-1}^{T} \operatorname{cov}_{q}^{-1} \mathbf{B}_{2 q-1} \\
\nu_{2 q-1}^{2} & =\frac{\sigma_{x}^{2}}{2}\left(\mu_{2 q-1}-\left(\mu_{2 q-1}\right)^{2}\right)
\end{aligned}
$$


and similarly the equations are also written for $\mu_{2 q}$ and $\nu_{2 q}^{2}$.

This assumption allows us to calculate the a posteriori and extrinsic information easily. As a result, the soft output MMSE detector with a priori information finds the estimated symbols $\hat{s}_{q}=\hat{\alpha}_{q}+j \hat{\beta}_{q}$ to compute the a posteriori L-values as

$L\left(u_{q r} \mid \hat{s}_{q}\right)=\ln \frac{\mathrm{P}\left(u_{q r}=+1\right)}{\mathrm{P}\left(u_{q r}=-1\right)}+\ln \frac{\sum_{\mathbf{u}_{q} \in \mathbb{L}_{q r,+1}} \mathrm{p}\left(\hat{s}_{q} \mid \mathbf{u}\right) \mathrm{P}\left(\mathbf{u} \mid u_{q r}\right)}{\sum_{\mathbf{u}_{q} \in \mathbb{L}_{q r,-1}} \mathrm{p}\left(\hat{s}_{q} \mid \mathbf{u}\right) \mathrm{P}\left(\mathbf{u} \mid u_{q r}\right)}$

for $q=1,2, \ldots, N_{t}$ and $r=1,2, \ldots, 2 M_{c}$ where $\mathbb{L}_{q r,+1}$ is the set of $2^{2 M_{c}-1}$ vectors $\mathbf{u}_{q}$ with $u_{q r}=+1$.

Then similar to (3), the a posteriori L-value is written as

$$
\begin{aligned}
L\left(u_{q r} \mid \mathbf{s}_{q}\right) & \approx \max _{\mathbf{u}_{q} \in \mathbb{L}_{q r,+1}}\left\{-\varrho_{q}+\sum_{r=1}^{2 M_{c}^{\prime}} \ln \mathrm{P}\left(u_{q r}\right)\right\} \\
& -\max _{\mathbf{u}_{q} \in \mathbb{L}_{q r,-1}}\left\{-\varrho_{q}+\sum_{r=1}^{2 M_{c}^{\prime}} \ln \mathrm{P}\left(u_{q r}\right)\right\}
\end{aligned}
$$

with

$$
\varrho_{q}=\frac{\left(\hat{\alpha}_{q}-\mu_{2 q-1} \lambda(u)\right)^{2}}{2 \nu_{2 q-1}^{2}}+\frac{\left(\hat{\beta}_{q}-\mu_{2 q} \lambda(u)\right)^{2}}{2 \nu_{2 q}^{2}}
$$

\section{Complexity Evaluation}

In this section, using the definition of the expected complexity exponent $c_{e}$, we evaluate the complexity evaluation for the breadth first $M$ and $T M$ algorithms and the soft output MMSE detector with a priori information. While evaluating the complexity of suboptimal detectors, we only count the flops of the main algorithm for the soft output MMSE detector without calculation cost of a priori information. For the breadth first detectors, we count the flops of both precomputing and main algorithm without taking into account for the cost of a priori information.

The expected complexity exponent $c_{e}$ of the algorithm is defined as

$$
c_{e}=\frac{\log C_{c}}{\log N_{t}}
$$

where $C_{c}$ is the expected complexity (average number of flops) per $N_{t}$ symbols and is calculated by counting the total number of elementary operations (addition, substraction, multiplication) [10].

Complexity exponent of $M$ and $T M$ detectors:

In [9], the complexity exponent $c_{e}$ of the sphere decoding has been derived as

$$
c_{e}=\frac{\log C_{c}\left(2 N_{t}, C_{0}^{2}\right)}{\log N_{t}}
$$

with the expected complexity

$$
C_{c}=\sum_{p=1}^{2 N_{t}} E_{c}\left(p, C_{0}^{2}\right) f_{c}(p)
$$

where $E_{c}$ is the average number of visited points at depth $p$ in $p$ dimensional sphere of radius $C_{0}$ and $f_{c}(p)=2\left(2 N_{t}-\right.$ $p+1)+17$ is the number of operations that the FinckePohst algorithm performs per each visited point at depth $p$. Therefore, the expected complexity is proportional to the number of visited points in the sphere of radius $C_{0}$.

For the $M$ algorithm, the average number of visited points at each depth is $E_{c}=2 M_{c} M_{b}$. Then, (30) becomes,

$C_{c}=2 M_{c} M_{b} \sum_{p=1}^{N_{d}}\left(2\left(2 N_{t}-p+1\right)+17\right)=2 M_{c} M_{b}\left(4 N_{t}^{2}+36 N_{t}\right)$

The complexity exponent of $M$ algorithm for $(4,4) \mathrm{V}$ BLAST considering different values of $M_{b}$ is listed in Table I.

\section{TABLE I}

THE COMPLEXITY EXPONENT OF $(4,4)$ V-BLAST USING $M$ ALGORITHM FOR THE DIFFERENT NUMBER OF CANDIDATES

\begin{tabular}{|c|c|c|c|c|c|c|}
\hline \hline$M_{b}$ & 1 & 2 & 4 & 8 & 16 & 32 \\
\hline \hline$c_{e}(\mathrm{~dB})(16 \mathrm{QAM})$ & 4.85 & 5.35 & 5.85 & 6.35 & 6.85 & 7.35 \\
\hline \hline$c_{e}(\mathrm{~dB})(\mathrm{QPSK})$ & 4.35 & 4.85 & 5.35 & 5.85 & 6.35 & 6.85 \\
\hline \hline
\end{tabular}

As a result, the complexity of main algorithm of the $M$ detector depends on the constellation size and the number of candidates and does not change according to noise variance. For the complexity of precomputing, we should add $2\left(2 N_{t}\right)^{3}$ for the QR decomposition. Thus, for example, the total expected complexity exponent of $(4,4)$ V-BLAST using $M$ algorithm with $M_{b}=16$ and $16 Q A M$ is 6.9 .

For the $T M$ algorithm, the number of visited points at each depth is not fixed as $M$ algorithm. Using the $T M$ algorithm with $C_{1}=15$ and $M_{b}=128$, for $(4,4)$ V-BLAST with QPSK, the complexity exponent is evaluated as $e_{c}=6.66$ considering precomputing process.

Moreover, the marginalization complexity of $M$ and $T M$ algorithms can be calculated as the number of maximization operations over $2 N_{t} M_{c} M_{b}$ possibilities.

Complexity exponent of the soft output MMSE detector:

In order to evaluate the overall complexity of the soft output MMSE detectors, the floating point operations (flops) are counted.

First, we evaluate the complexity of (19) as $N_{d}^{3}+2 N_{d}^{2}+N_{d}$ where $N_{d}=2 N_{t}$. In order to get the inversion of (19), we use the QR decomposition and we count it as $3 N_{d}^{3}+N_{d}^{2}+$ $N_{d} / 3$. The recursive methods as described in [5] could be used to reduce this complexity. Then, using (19), we calculate the complexity of (17) and (18). While equation (17) requires $N_{d}^{2}+3 N_{d}$, equation (18) needs only $2 N_{d}$. In totally, we obtain $N_{d}^{2}+5 N_{d}$ flops for them. The last part is the calculation of the complexity of mean and variances given by (23) and (24) which require $4 N_{d}+6$. As a result, the total complexity evaluation for the soft output MMSE detector considering all $2 M_{c} N_{t}$ bits is evaluated as $\left(4 N_{d}^{3}+4 N_{d}^{2}+10 N_{d}+N_{d} / 3+6\right) N_{t}$ flops.

For $(4,4)$ V-BLAST using MMSE detector, the expected complexity exponent is calculated as 6.61. 
Moreover, the marginalization complexity of the soft output MMSE detector is calculated as the number of maximization operations over $2 N_{t} M_{c} 2^{2 M c}$ possibilities.

When the number of constellation points $\left(2^{2 M c}\right)$ is equal to the number of candidates $\left(M_{b}\right)$, the marginalization complexity of the breadth first $M$ and $T M$ algorithms is almost the same as the soft output MMSE detector.

\section{Simulation Results}

In this section, we give the simulation results for $(4,4) \mathrm{V}$ BLAST for independent Rayleigh fading channels assuming the channel state information is available at the receiver. We use a simple rate $1 / 2(7,5) \mathrm{CC}$ as outer code with 16QAM $\left(M_{c}=2\right)$ and QPSK $\left(M_{c}=1\right)$ which provide us the rate of $8 \mathrm{bps} / \mathrm{Hz}$ and $4 \mathrm{bps} / \mathrm{Hz}$ respectively.

Figure 2 compares the bit-error-rate (BER) performance of the list $M$ detector with $M_{b}=16$ and the soft output MMSE detector using 16QAM and 4 iterations. For almost the same complexity, both detectors give almost the same performance for the first and second iterations at $8 \mathrm{bps} / \mathrm{Hz}$. However, for the third and fourth iterations, the soft output MMSE receiver gives better performance than list $M$ detector with $M_{b}=16$ at $8 \mathrm{bps} / \mathrm{Hz}$. In order to obtain the same performance, we should increase the number of candidates for the $M$ algorithm which causes an increase in the complexity.

In Figure 3, the comparison results for the soft output MMSE detector and the list $T M$ detector with $C_{1}=15$ and $M_{b}=128$ using QPSK and 4 iterations are illustrated. For almost the same complexity, the breadth first $T M$ algorithm gives better performance for first two iterations at $4 \mathrm{bps} / \mathrm{Hz}$.

\section{CONCLUSION}

In this paper, we have compared the soft output MMSE detector with a priori information and the breadth first $M$ and $T M$ algorithms. We have shown that at almost the same complexity, the soft output MMSE gives better performance than the $M$ detector at $8 \mathrm{bps} / \mathrm{Hz}$. Furthermore, we have shown that for almost the same complexity, the $T M$ detector converges faster than the soft output MMSE detector at $4 \mathrm{bps} / \mathrm{Hz}$. The complexity analysis just gives order of complexity and should be completed by taking into account the hardware constraints.

\section{REFERENCES}

[1] E. Telatar, "Capacity of multiple antenna Gaussian channels". AT\&T Bell Laboratories Technical Report, 1995.

[2] G. J. Foschini, "Layered space time architecture for wireless communication in a fading environment when using multi-element antennas", Bell Labs Tech. Journal vol. 1,pp. 41-59, 1996.

[3] J. J. Boutros and F. Boixadera and C. Lamy, "Bit interleaved coded modulation for multiple input multiple output channels", Proceedings of IEEE ISSTA, vol. 1, pp. 123-126, 2000.

[4] B. Hochwald and S. Ten Brink, "Achieving near capacity on a multiple antenna channel", IEEE Transaction on Communication, vol. 51, pp. 389399, 2003.

[5] X. Wang and V. Poor, "Iterative (turbo) soft interference cancellation and decoding for coded CDMA", IEEE Transaction on Communications, vol. 47,pp. 1046-1060, 1999.

[6] A. Guegen, "Comparison of suboptimal iterative space-time receivers", Proceedings of IEEE VTC-Spring, pp. 842-846, 2003.

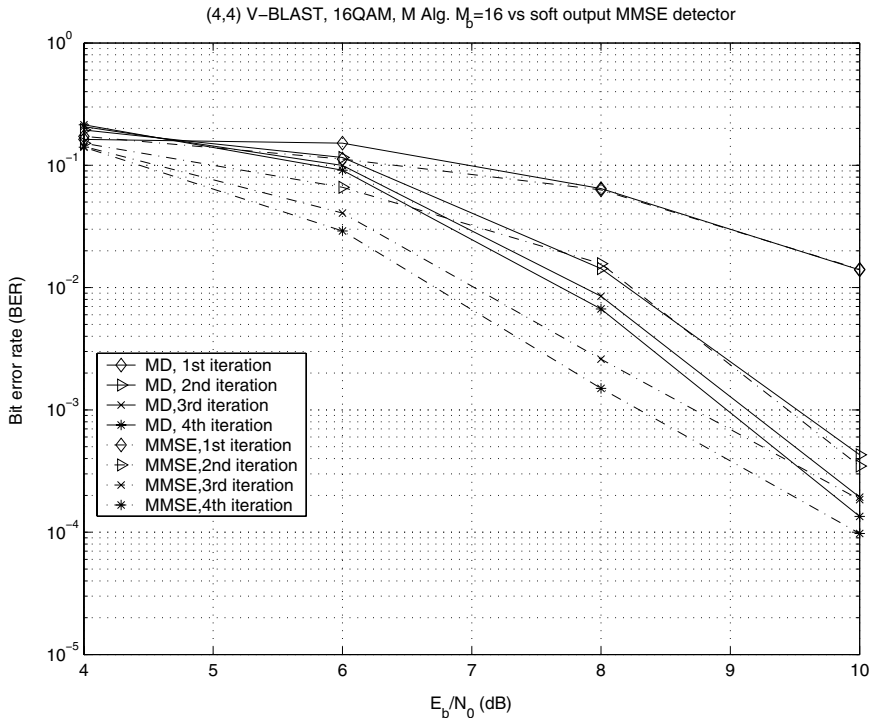

Fig. 2. The BER comparison of the soft output MMSE and the breadth first $M$ detector (MD) with $M_{b}=16$ for 16QAM at the same complexity exponent

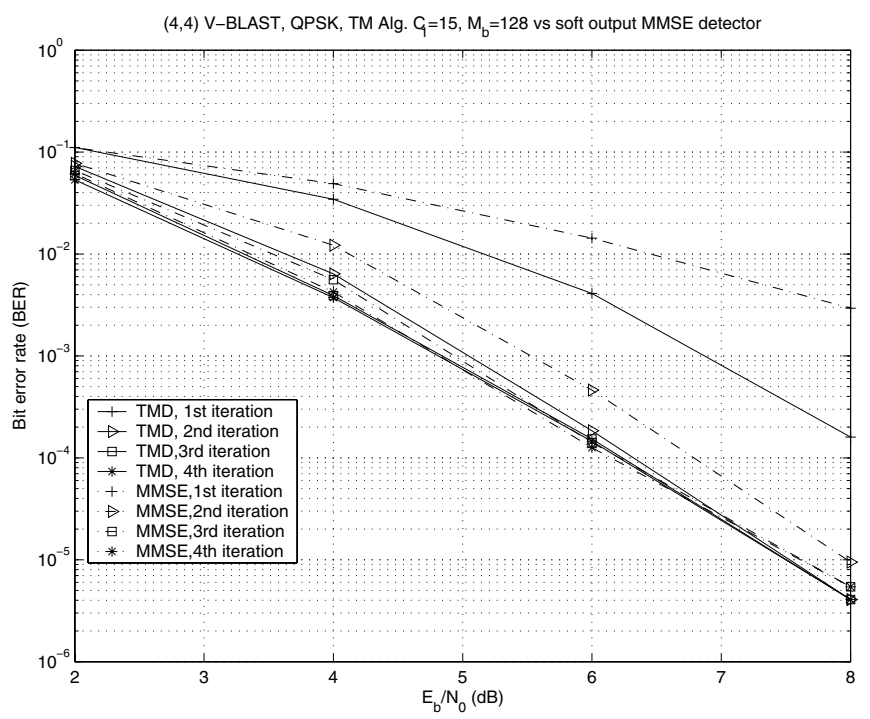

Fig. 3. The BER comparison of the soft output MMSE and the breadth first $T M$ detector (TMD) with $C_{1}=15$ and $M_{b}=128$ for QPSK at the same complexity exponent

[7] D. Le Ruyet, T. Bertozzi, B. Ozbek, "Breadth first algorithms for APP detectors over MIMO channels", Proceedings of IEEE ICC, pp. 926-930, June 2004.

[8] M. Tuchler and A. Singer and R. Koetter, "Minimum mean squared error equalization using a priori information", IEEE Transaction on Signal Proccessing, vol.50, pp. 673-683, 2002.

[9] B. Hassibi and H. Vikalo, "On sphere decoding algorithm. 1. Expected complexity", To appear in IEEE Transactions on Signal Processing, 2004.

[10] G. H. Golub and C. F. Van Loan, "Matrix Computations, 3rd edition", The Johns Hopkins University Press, Baltimore and London, 1996. 